FACTA UNIVERSITATIS (NIŠ)

Ser. Math. Inform. Vol. 32, No 1 (2017), 117-128

DOI:10.22190/FUMI1701117P

\title{
ON THE $\mathcal{M}$-PROJECTIVE CURVATURE TENSOR OF A $(k, \mu)$-CONTACT METRIC MANIFOLD *
}

\author{
D. G. Prakasha and Kakasab Mirji
}

\begin{abstract}
The paper deals with the study of $\mathcal{M}$-projective curvature tensor on $(k, \mu)$ contact metric manifolds. We classify non-Sasakian $(k, \mu)$-contact metric manifold satisfying the conditions $R(\xi, X) \cdot \mathcal{M}=0$ and $\mathcal{M}(\xi, X) \cdot S=0$, where $R$ and $S$ are the Riemannian curvature tensor and the Ricci tensor, respectively. Finally, we prove that a $(k, \mu)$-contact metric manifold with vanishing extended $\mathcal{M}$-projective curvature tensor $\mathcal{M}^{e}$ is a Sasakian manifold.

Keywords: $(k, \mu)$-contact metric manifold, $N(k)$-contact metric manifold, Sasakian manifold, $\mathcal{M}$-projective curvature tensor, Einstein manifold.
\end{abstract}

\section{Introduction}

The unit tangent sphere bundle of a Riemannian manifold of constant sectional curvature tensor admits a contact metric structure $(\varphi, \xi, \eta, g)$ such that the characteristic vector field $\xi$ belongs to the $(k, \mu)$-nullity distribution for some real numbers $k$ and $\mu$. This means that for any vector fields $X$ and $Y$ the curvature tensor $R$ satisfies the condition

$$
R(X, Y) \xi=(k I+\mu h)(\eta(Y) X-\eta(X) Y),
$$

and $h$ denote Lie derivative of the structure tensor field $\varphi$ in the direction of $\xi$. This class of contact metric manifolds which satisfies (1.1) were introduced by Blair, Koufogiorgos and Papantoniou [4]. The class of $(k, \mu)$-contact metric manifolds encloses both Sasakian and non-Sasakian manifolds. A full classification of $(k, \mu)$-contact metric manifolds was given by Boeckx [6]. $(k, \mu)$-contact metric manifolds are invariant under $D$-homothetic transformations. In [8], Cho studied a

Received May 09, 2016.; accepted September 14, 2016.

2010 Mathematics Subject Classification. Primary 53C25; Secondary 53D15

*The first author is thankful to UGC, New Delhi for financial support in the form of UGC-SAPDRS-III programme to the Department of Mathematics, Karnatak University, Dharwad, India. Also, the second author is thankful to Karnatak University, Dharwad for financial support in the form of UGC-UPE fellowship. 
conformally flat contact Riemannian $(k, \mu)$-space and such a space with vanishing C-Bochner curvature tensor. Subsequently many geometers studied the contact metric manifold with different curvature tensors and obtained various important properties. Conformal curvature tensor of a $(k, \mu)$-contact metric manifold was studied in the papers [9] and [13]. In a paper [5], Blair, Kim and Tripathi started to study of concircular curvature tensor of a contact metric manifolds. The study of pseudo projective curvature tensor on a contact metric manifold was found in [1]. Recently, quasi-conformal curvature tensor and $E$-Bochner curvature tensor on a $(k, \mu)$-contact metric manifold has been studied respectively in the papers [10] and [12]. Apart from the Riemannian curvature tensor, Weyl conformal curvature tensor and concircular curvature tensor, the $\mathcal{M}$-projective curvature tensor is another important tensor from the differential geometric point of view. This curvature tensor bridges the gap between conformal curvature tensor, conharmonic curvature tensor and concircular curvature tensor on one side and $H$-projective curvature tensor on the other. The $\mathcal{M}$-projective curvature tensor $\mathcal{M}$ on a $(2 n+1)$-dimensional Riemannian manifold is given by [17]

$$
\begin{aligned}
\mathcal{M}(X, Y) Z= & R(X, Y) Z-\frac{1}{4 n}[S(Y, Z) X-S(X, Z) Y \\
& +g(Y, Z) Q X-g(X, Z) Q Y],
\end{aligned}
$$

where $R$ is the Riemannian curvature tensor, $S$ is the Ricci curvature tensor and $Q$ is the Ricci operator defined by $g(Q X, Y)=S(X, Y)$, for any $X, Y \in M$. Some properties of this tensor had been studied earlier in $([7,11,14,15])$ and many others.

The paper is organized as follows. Section 2 deals with some preliminaries on $(k, \mu)$-contact metric manifold. In section 3 , we study non-Sasakian $(k, \mu)$-contact metric manifold satisfying $R(\xi, X) \cdot \mathcal{M}=0$. In section 4 , we classify the nonSasakian $(k, \mu)$-contact metric manifold satisfying $\mathcal{M}(\xi, X) \cdot S=0$. The last section is devoted to study $(k, \mu)$-contact metric manifold with vanishing extended $\mathcal{M}$ projective curvature tensor.

\section{Preliminaries}

A $(2 n+1)$-dimensional differentiable manifold $M$ is called an almost contact manifold if either its structural group can be reduced to $U(n) \times 1$ or equivalently, there is an almost contact structure $(\varphi, \xi, \eta)$ consisting of a $(1,1)$ tensor field $\varphi$, a vector field $\xi$, and a 1-form $\eta$ satisfying

$$
\begin{gathered}
\varphi^{2} X=-X+\eta(X) \xi, \\
\eta(\xi)=1, \quad \varphi \xi=0, \quad \eta \cdot \varphi=0 .
\end{gathered}
$$

An almost contact structure is said to be normal if the induced almost complex structure $J$ on the product manifold $M \times \mathbb{R}$ defined by

$$
J\left(X, \lambda \frac{d}{d t}\right)=\left(\varphi X-\lambda \xi, \eta(X) \frac{d}{d t}\right)
$$


is integrable, where $X$ is tangent to $M, t$ the coordinate of $\mathbb{R}$ and $\lambda$ a smooth function on $M \times \mathbb{R}$. The condition for being normal is equivalent to vanishing of the torsion tensor $[\varphi, \varphi]+2 d \eta \otimes \xi$, where $[\varphi, \varphi]$ is the Nijenhuis tensor of $\varphi$. Let $g$ be a compatible Riemannian metric with $(\varphi, \xi, \eta)$, that is,

$$
g(\varphi X, \varphi Y)=g(X, Y)-\eta(X) \eta(Y)
$$

or equivalently,

$$
g(X, \varphi Y)=-g(\varphi X, Y) \quad \text { and } \quad g(X, \xi)=\eta(X),
$$

for all vector fields $X, Y$. Then, $M$ become an almost contact metric manifold equipped with an almost contact metric structure $(\varphi, \xi, \eta, g)$.

An almost contact metric structure becomes a contact metric structure if

$$
g(X, \varphi Y)=d \eta(X, Y) \text {, for all vector fields } X, Y \text { on } M .
$$

In a contact metric manifold, the $(1,1)$-tensor field $h$ is symmetric and satisfies (2.4) $h \xi=0, \quad h \varphi=-\varphi h, \quad \nabla \xi=-\varphi-\varphi h, \quad \operatorname{trace}(h)=\operatorname{trace}(\varphi h)=0$.

A normal contact metric manifold is a Sasakian manifold [2]. An almost contact metric manifold is Sasakian if and only if

$$
\left(\nabla_{X} \varphi\right) Y=g(X, Y) \xi-\eta(Y) X,
$$

while a contact metric manifold $M$ is Sasakian if and only if

$$
R(X, Y) \xi=\eta(Y) X-\eta(X) Y, \text { for all vector fields } X, Y \text { on } M .
$$

The $(k, \mu)$-nullity distribution $N(k, \mu)$ for a contact metric manifold $M$ for the pair $(k, \mu) \in \mathbb{R}^{2}$, is a distribution $([4,16])$

$$
\begin{aligned}
N(k, \mu) \quad: \quad P \longmapsto N_{P}(k, \mu) \\
=\left\{U \in T_{P} M \mid R(X, Y) U=(k I+\mu h)(g(Y, Z) X-g(X, Z) Y), \forall X, Y \in T_{P} M\right\} .
\end{aligned}
$$

A contact metric manifold with $\xi \in N(k, \mu)$ is called a $(k, \mu)$-manifold. For a $(k, \mu)$-manifold it is known that $h^{2}=(k-1) \varphi^{2}$. This class contains Sasakian manifolds for $k=1$ and $h=0$. In fact, for $(k, \mu)$ - manifold the condition of being Sasakian manifold, $K$-contact manifold, $k=1$ and $h=0$ are all equivalent. If $\mu=0$, the $(k, \mu)$-nullity distribution $N(k, \mu)$ is reduced to the $k$-nullity distribution $N(k)$ [18]. Further if $\xi$ belongs to $N(k)$, then we call a contact metric manifold $M$ an $N(k)$-contact metric manifold.

In a $(k, \mu)$-contact metric manifold the following relations hold [4]:

$$
\begin{aligned}
h^{2} & =(k-1) \varphi^{2}, \quad k \leq 1, \\
R(\xi, X) Y & =k[g(X, Y) \xi-\eta(Y) X]+\mu[g(h X, Y) \xi-\eta(Y) h X], \\
S(X, \xi) & =2 n k \eta(X), \\
S(X, Y) & =[2(n-1)-n \mu] g(X, Y)+[2(n-1)+\mu] g(h X, Y) \\
& +[2(1-n)+n(2 k+\mu)] \eta(X) \eta(Y), \\
r & =2 n[2 n-2+k-n \mu],
\end{aligned}
$$


for all vector fields $X, Y$ on $M$, where $S$ is the Ricci tensor of type $(0,2), Q$ is the Ricci operator, i.e., $S(X, Y)=g(Q X, Y)$ and $r$ is the scalar curvature of the manifold.

From (1.1) and (1.2), it can be easily verified that, on a $(k, \mu)$-contact metric manifold the $\mathcal{M}$-projective curvature tensor takes the following forms:

(2.15) $g\left(\mathcal{M}\left(e_{i}, Y\right) Z, e_{i}\right)=\left(\frac{2 n+1}{4 n}\right) S(Y, Z)-\left(\frac{r}{4 n}\right) g(Y, Z)$,

for all vector fields $X, Y$ and $Z$ on $M$, where $\left\{e_{i}\right\}, i=1,2, \ldots(2 n+1)$ be an orthonormal basis of the tangent space.

We recall the following theorem on contact metric manifold due to Blair:

Lemma 2.1. [3] A contact metric manifold $M^{2 n+1}$ satisfying $R(X, Y) \xi=0$ is locally isometric to $E^{n+1}(0) \times S^{n}(4)$ for $n>1$ and flat for $n=1$.

In general, in a $(k, \mu)$-contact metric manifold the Ricci operator $Q$ does not commutes with $\varphi$. However, Yildiz and De proved the following:

Lemma 2.2. [19] In a non-Sasakian $(k, \mu)$-contact metric manifold the following conditions are equivalent:

(i) $\eta$-Einstein manifold,

(ii) $Q \varphi=\varphi Q$.

Corollary 2.1. [19] A 3-dimensional non-Sasakian $(k, \mu)$-contact $\eta$-Einstein manifold is an $N(k)$-contact metric manifold. 
Definition 2.1. A $(k, \mu)$-contact metric manifold $M$ is said to be $\eta$-Einstein if the Ricci operator $Q$ satisfies

$$
Q=\alpha I+\beta \eta \otimes \xi
$$

where $\alpha$ and $\beta$ are smooth functions on the manifold. In particular if $\beta=0$, then $M$ is an Einstein manifold.

\section{3. $(k, \mu)$-contact metric manifold satisfying $R(\xi, X) \cdot \mathcal{M}=0$}

Let us consider that $M$ be a $(2 n+1)$-dimensional $(k, \mu)$-contact metric manifold which satisfies $R(\xi, X) \cdot \mathcal{M}=0$.

Therefore $R(\xi, X) \cdot \mathcal{M}(Y, Z) W=0$ implies that

$$
\begin{aligned}
& R(\xi, X) \mathcal{M}(Y, Z) W-\mathcal{M}(R(\xi, X) Y, Z) W-\mathcal{M}(Y, R(\xi, X) Z) W \\
& -\mathcal{M}(Y, Z) R(\xi, X) W=0 .
\end{aligned}
$$

By virtue of (2.6) and (3.1), we have

$$
\begin{aligned}
& k[\eta(\mathcal{M}(Y, Z) W) X-g(X, \mathcal{M}(Y, Z) W) \xi-\eta(Y) \mathcal{M}(X, Z) W \\
& +g(X, Y) \mathcal{M}(\xi, Z) W-\eta(Z) \mathcal{M}(Y, X) W+g(X, Z) \mathcal{M}(Y, \xi) W \\
& -\eta(W) \mathcal{M}(Y, Z) X+g(X, W) \mathcal{M}(Y, Z) \xi] \\
& +\mu[\eta(\mathcal{M}(Y, Z) W) h X-g(h X, \mathcal{M}(Y, Z) W \xi-\eta(Y) \mathcal{M}(h X, Z) W \\
& +g(h X, Y) \mathcal{M}(\xi, Z) W-\eta(Z) \mathcal{M}(Y, h X) W+g(h X, Z) \mathcal{M}(Y, \xi) W \\
& -\eta(W) \mathcal{M}(Y, Z) h X+g(h X, W) \mathcal{M}(Y, Z) \xi]=0 .
\end{aligned}
$$

Putting $W=\xi$ in $(3.2)$, we have

$$
\begin{aligned}
& k[\eta(\mathcal{M}(Y, Z) \xi) X-g(X, \mathcal{M}(Y, Z) \xi) \xi-\eta(Y) \mathcal{M}(X, Z) \xi \\
& +g(X, Y) \mathcal{M}(\xi, Z) \xi-\eta(Z) \mathcal{M}(Y, X) \xi+g(X, Z) \mathcal{M}(Y, \xi) \xi \\
& -\mathcal{M}(Y, Z) X+\eta(X) \mathcal{M}(Y, Z) \xi] \\
& +\mu[\eta(\mathcal{M}(Y, Z) \xi) h X-g(h X, \mathcal{M}(Y, Z) \xi) \xi-\eta(Y) \mathcal{M}(h X, Z) \xi \\
& +g(h X, Y) \mathcal{M}(\xi, Z) \xi-\eta(Z) \mathcal{M}(Y, h X) \xi \\
& +g(h X, Z) \mathcal{M}(Y, \xi) \xi-\mathcal{M}(Y, Z) h X]=0 .
\end{aligned}
$$

By substituting the value of $(2.10)-(2.14)$ in (3.3), we obtain

$$
\text { (3.4) } \begin{aligned}
& \frac{k}{4 n}[\{2(1-n)+n(2 k+\mu)\}(g(X, Z) Y-g(X, Y) Z) \\
& +\{2(1-n)+\mu(4 n-1)\}(g(X, Z) h Y-g(X, Y) h Z \\
& +g(h Z, X) \eta(Y) \xi-g(h Y, X) \eta(Z) \xi)] \\
& +\frac{\mu}{4 n}[\{2(1-n)+n(2 k+\mu)\}(g(h X, Z) Y-g(h X, Y) Z) \\
& +\{2(1-n)+\mu(4 n-1)\}(g(h X, Z) h Y-g(h X, Y) h Z \\
& +g(h Z, h X) \eta(Y) \xi-g(h Y, h X) \eta(Z) \xi)]-k \mathcal{M}(Y, Z) X-\mu \mathcal{M}(Y, Z) h X=0 .
\end{aligned}
$$


Taking the inner product of (3.4) with $U$, we have

$$
\begin{aligned}
& \frac{k}{4 n}[\{2(1-n)+n(2 k+\mu)\}(g(X, Z) g(Y, U)-g(X, Y) g(Z, U) \\
& +\{2(1-n)+\mu(4 n-1)\}(g(X, Z) g(h Y, U)-g(X, Y) g(h Z, U) \\
& +g(h Z, X) \eta(Y) \eta(U)-g(h Y, X) \eta(Z) \eta(U))] \\
& +\frac{\mu}{4 n}[\{2(1-n)+n(2 k+\mu)\}(g(h X, Z) g(Y, U)-g(h X, Y) g(Z, U)) \\
& +\{2(1-n)+\mu(4 n-1)\}(g(h X, Z) g(h Y, U)-g(h X, Y) g(h Z, U) \\
& +g(h Z, h X) \eta(Y) \eta(U)-g(h Y, h X) \eta(Z) \eta(U))] \\
& -k g(\mathcal{M}(Y, Z) X, U)-\mu g(\mathcal{M}(Y, Z) h X, U)=0 .
\end{aligned}
$$

Let $\left\{e_{i}\right\}, i=1,2, \ldots,(2 n+1)$ be an orthonormal basis of the tangent space. Putting $Y=U=e_{i}$ in (3.5) and taking the summation over $i$ and using (2.15), we have

$$
\begin{aligned}
& {[2 n k\{2(1-n)+n(2 k+\mu)\}+k r] g(X, Z)} \\
& +[2 n \mu\{2(1-n)+n(2 k+\mu)\}+\mu r] g(h X, Z) \\
& -k(2 n+1) S(X, Z)-\mu(2 n+1) S(h X, Z)=0 .
\end{aligned}
$$

In view of (2.8) and (2.9), (3.6) takes the form

$$
\begin{aligned}
& g(h X, Z) \\
& =\left[\frac{k(2(n-1)-n(2 k+\mu))-\mu(k-1)(2(n-1)+\mu)}{\mu(2(1-n)+n(2 k+\mu))-k(2(n-1)+\mu)}\right] g(X, Z) \\
& +\left[\frac{k(2(1-n)+n(2 k+\mu))+\mu(k-1)(2(n-1)+\mu)}{\mu(2(1-n)+n(2 k+\mu))-k(2(n-1)+\mu)}\right] \eta(X) \eta(Z) .
\end{aligned}
$$

By virtue of (2.8) and (3.7), we have the following:

$$
S(X, Z)=a g(X, Z)+b \eta(X) \eta(Z),
$$

where

$$
\begin{aligned}
a & =[2(n-1)-n \mu] \\
& +\frac{(2(n-1)+\mu)[k(2(n-1)-n(2 k+\mu))-\mu(k-1)(2(n-1)+\mu)]}{\mu(2(1-n)+n(2 k+\mu))-k(2(n-1)+\mu)}
\end{aligned}
$$

and

$$
\begin{aligned}
b & =[2(1-n)+n(2 k+\mu)] \\
& +\frac{(2(n-1)+\mu)[k(2(1-n)+n(2 k+\mu))+\mu(k-1)(2(n-1)+\mu)]}{\mu(2(1-n)+n(2 k+\mu))-k(2(n-1)+\mu)} .
\end{aligned}
$$

Thus from (3.8), we can state the following:

Theorem 3.1. $A(2 n+1)$-dimensional $(k, \mu)$-contact metric manifold $M$ satisfying $R(\xi, X) \cdot \mathcal{M}=0$ is an $\eta$-Einstein manifold. 
From the Lemma 2.2, we can state the following:

Corollary 3.1. Let $M$ be a $(2 n+1)$-dimensional non-Sasakian $(k, \mu)$-contact metric manifold satisfying $R(\xi, X) \cdot \mathcal{M}=0$. Then the Ricci operator $Q$ commutes with $\varphi$.

From the Corollary 2.1, we can state the following:

Corollary 3.2. A 3-dimensional non-Sasakian $(k, \mu)$-contact $\eta$-Einstein manifold $M$ satisfying $R(\xi, X) \cdot \mathcal{M}=0$ is an $N(k)$-contact metric manifold.

4. $(k, \mu)$-contact metric manifold satisfying $\mathcal{M}(\xi, X) \cdot S=0$

Let $M$ be a $(2 n+1)$-dimensional $(k, \mu)$-contact metric manifold satisfying condition $\mathcal{M}(\xi, X) \cdot S=0$.

Therefore, $(\mathcal{M}(\xi, X) \cdot S)(Y, Z)=0$ implies that

$$
S(\mathcal{M}(\xi, X) Y, Z)+S(Y, \mathcal{M}(\xi, X) Z)=0 .
$$

Putting $Z=\xi$ in (4.1), we have

$$
S(\mathcal{M}(\xi, X) Y, \xi)+S(Y, \mathcal{M}(\xi, X) \xi)=0
$$

In view of (2.11) and (2.12), (2.7) gives

$$
\begin{aligned}
(4.3) S(\mathcal{M}(\xi, X) Y, \xi) & =2 n k\left(\frac{2(1-n)+n(2 k+\mu)}{4 n}\right)(g(X, Y)-\eta(X) \eta(Y)) \\
& +2 n k\left(\frac{2(1-n)+\mu(4 n-1)}{4 n}\right) g(h X, Y)
\end{aligned}
$$

and

$$
\begin{aligned}
(4.4) S(Y, \mathcal{M}(\xi, X) \xi) & =\left(\frac{2(1-n)+n(2 k+\mu)}{4 n}\right)(2 n k \eta(X) \eta(Y)-S(X, Y)) \\
& -\left(\frac{2(1-n)+\mu(4 n-1)}{4 n}\right) S(h X, Y)
\end{aligned}
$$

respectively.

Substituting (4.3) and (4.4) in (4.2) followed by a simple calculation gives,

$$
\begin{aligned}
& (2(1-n)+n(2 k+\mu))[S(X, Y)-2 n k g(X, Y)] \\
& +(2(1-n)+\mu(4 n-1))[S(h X, Y)-2 n k g(h X, Y)]=0 .
\end{aligned}
$$

By virtue of (2.3), (2.4), (2.5) and (2.8), we have

$$
\begin{aligned}
S(h X, Y) & =(2(n-1)-n \mu) g(h X, Y) \\
& -(k-1)(2(n-1)+\mu)(g(X, Y)-\eta(X) \eta(Y)) .
\end{aligned}
$$


From (2.8) and (2.16), one can see that a non-Sasakian $(k, \mu)$-contact metric manifold $M$ is an $\eta$-Einstein if and only if $\mu=-2(n-1)$. In this case the Ricci tensor $S$ is given by

$$
S(X, Y)=2\left(n^{2}-1\right) g(X, Y)-2\left(n^{2}-n k-1\right) \eta(X) \eta(Y) .
$$

Putting $\mu=-2(n-1)$ in $(2.9)$, we obtain

$$
r=2 n(k+2(n-1)(n+1)) .
$$

Now by considering $\mu=-2(n-1)$ in $(4.3)$, then it takes the form

$$
\begin{aligned}
S(\mathcal{M}(\xi, X) Y, \xi) & =4 n k\left(\frac{1+n k-n^{2}}{4 n}\right)(g(X, Y)-\eta(X) \eta(Y)) \\
& +\left(\frac{16 n^{2} k(1-n)}{4 n}\right) g(h X, Y) .
\end{aligned}
$$

In view of (4.4) and (4.7), we get

$$
\begin{aligned}
(4.10) S(Y, \mathcal{M}(\xi, X) \xi) & =4\left(n^{2}-1\right)\left(\frac{1+n k-n^{2}}{4 n}\right)(\eta(X) \eta(Y)-g(X, Y)) \\
& -\left(\frac{16 n(1-n)\left(n^{2}-1\right)}{4 n}\right) g(h X, Y) .
\end{aligned}
$$

From (4.2), (4.9) and (4.10), we get

$4\left(1+n k-n^{2}\right)^{2}(g(X, Y)-\eta(X) \eta(Y))+16 n(1-n)\left(1+n k-n^{2}\right) g(h X, Y)=0$.

Contracting the above equation and then by taking account of (2.4), we have

$$
8 n\left(1+n k-n^{2}\right)^{2}=0 .
$$

This implies

$$
k=\frac{n^{2}-1}{n}
$$

which is equivalent to $\left(1+n k-n^{2}\right)=0$. Thus in view of (4.7), $M$ reduces to an Einstein manifold. Hence we state the following:

Theorem 4.1. In a $(2 n+1)$-dimensional non-Sasakian $\eta$-Einstein $(k, \mu)$-contact metric manifold $M$ if the $\mathcal{M}$-projective curvature tensor $\mathcal{M}$ satisfies $\mathcal{M}(\xi, X) \cdot S=0$, then $M$ reduces to an Einstein manifold.

From (4.11), we have $k=\left(n^{2}-1\right) / n<1$. So $n=1$ is the only case. This gives $\mu=0$ which with $n=1$ gives $k=0$. Thus substituting $k=0=\mu$ in (1.1), we state the following: 
Theorem 4.2. In a $(2 n+1)$-dimensional non-Sasakian $\eta$-Einstein $(k, \mu)$-contact metric manifold $M$ if the $\mathcal{M}$-projective curvature tensor $\mathcal{M}$ satisfies $\mathcal{M}(\xi, X) \cdot S=0$, then $M$ is flat and 3-dimensional.

Next, let $M$ be a $(2 n+1)$-dimensional $(k, \mu)$-contact metric manifold satisfying $\mathcal{M}(\xi, X) \cdot S=0$. Then we have the following four possible cases.

Case-1: Suppose $k=0=\mu$.

From (1.1), we have $R(X, Y) \xi=0$. Thus, in view of Lemma 2.1, $M$ is flat and 3 -dimensional or it is locally isometric to $E^{n+1}(0) \times S^{n}(4)$.

Case-2: Suppose $k \neq 0=\mu$.

Using $\mu=0$ in (4.5), we have $S(X, Y)=2 n k g(X, Y)$. Thus $M$ reduces to an Einstein Sasakian manifold.

Case-3(i): Suppose $k=0 \neq \mu$ and $n>1$.

Using $k=0$ in (4.5), (2.8) and (4.6), we get

$$
\begin{aligned}
(2(n-1)-n \mu) S(X, Y) & =(2(1-n)+\mu(4 n-1)) S(h X, Y), \\
S(X, Y) & =(2(n-1)-n \mu)(g(X, Y)-\eta(X) \eta(Y)) \\
& +(2(n-1)+\mu) g(h X, Y) \text { and } \\
S(h X, Y) & =(2(n-1)-n \mu) g(h X, Y) \\
& +(2(n-1)+\mu)(g(X, Y)-\eta(X) \eta(Y))
\end{aligned}
$$

respectively.

From the above three equations, we get $S(X, Y)=C[g(X, Y)-\eta(X) \eta(Y)]$, for some suitable $C$. Now in view of Theorem 4.2, we see that the Case-3(i) is not possible.

Case-3(ii): Suppose $k=0 \neq \mu$ and $n=1$.

Using $k=0$ and $n=1$ in (4.5), (2.8) and (4.6), we get

$$
\begin{aligned}
\mu S(X, Y) & =-3 \mu S(h X, Y) \\
S(X, Y) & =-\mu(g(X, Y)-\eta(X) \eta(Y))+\mu g(h X, Y) \text { and } \\
S(h X, Y) & =-\mu g(h X, Y)+\mu(g(X, Y)-\eta(X) \eta(Y))
\end{aligned}
$$

respectively.

From the above three relations, we get $2 \mu S(X, Y)=0$. This gives either $\mu=0$ or $S(X, Y)=0$. If $\mu=0$ which is a contradiction.

If $S(X, Y)=0$, then taking $X=Y=\xi$, we have

$$
S(\xi, \xi)=2 n k=0
$$

which implies that $k=0$. Using $k=0$ in $(2.9)$, we get $n \mu=2(n-1)$. But we have $n=1$, this implies $\mu=0$, which is a contradiction. Thus, Case-3(ii) is also not possible.

Case-4(i): Suppose $k \neq 0, \mu \neq 0$ and $n>1$.

After eliminating $g(h X, Y)$ and $S(h X, Y)$ from (4.5), (2.8) and (4.6), we get $S(X, Y)=$ $\alpha g(X, Y)+\beta \eta(X) \eta(Y)$, for some suitable $\alpha$ and $\beta$. Thus $M$ reduces to an $\eta$-Einstein manifold. 
Case-4(ii): Suppose $k \neq 0, \mu \neq 0$ and $n=1$.

Putting $n=1$ in (4.5), (2.8) and (4.6), we get

$$
\begin{aligned}
(2 k+\mu) S(X, Y) & =2 k(2 k+\mu) g(X, Y)+6 k \mu g(h X, Y)-3 \mu S(h X, Y), \\
S(X, Y) & =-\mu g(X, Y)+\mu g(h X, Y)+(2 k+\mu) \eta(X) \eta(Y) \text { and } \\
S(h X, Y) & =-\mu g(h X, Y)-(k-1) \mu g(X, Y)+(k-1) \mu \eta(X) \eta(Y)
\end{aligned}
$$

respectively.

Eliminating $g(h X, Y)$ and $S(h X, Y)$ from the above three equations, we have $S(X, Y)=$ $\alpha g(X, Y)+\beta \eta(X) \eta(Y)$, for some suitable $\alpha$ and $\beta$. Thus, $M$ is an $\eta$-Einstein manifold and in this case $\mu=-2(n-1)$. But $n=1$, implies $\mu=0$ which is a contradiction. Hence this case is not possible. Thus from the above four possible cases, we can able to state the following:

Theorem 4.3. Let $M$ be a $(2 n+1)$-dimensional non-Sasakian $(k, \mu)$-contact metric manifold satisfying $\mathcal{M}(\xi, X) \cdot S=0$. Then we have one of the following:

(i) $M$ is flat and 3-dimensional;

(ii) $M$ is locally isometric to $E^{n+1}(0) \times S^{n}(4)$;

(iii) $M$ is an $\eta$-Einstein manifold;

(iv) $M$ is a 3-dimensional Einstein manifold.

\section{5. $(k, \mu)$-contact metric manifold with vanishing extended $\mathcal{M}$-projective curvature tensor}

The extended $\mathcal{M}$-projective curvature tensor $\mathcal{M}^{e}$ of $\mathcal{M}$-projective curvature tensor $\mathcal{M}$ is defined as follows:

$$
\begin{aligned}
\mathcal{M}^{e}(X, Y) Z & =\mathcal{M}(X, Y) Z-\eta(X) \mathcal{M}(\xi, Y) Z \\
& -\eta(Y) \mathcal{M}(X, \xi) Z-\eta(Z) \mathcal{M}(X, Y) \xi
\end{aligned}
$$

Putting $Y=Z=\xi$ and supposing that the extended $\mathcal{M}$-projective curvature tensor vanishes, we get from (5.1)

$$
\mathcal{M}(X, \xi) \xi=0
$$

By virtue of (2.13) and (5.2), we obtain

$$
\begin{aligned}
& {\left[\frac{(2(1-n)+n(2 k+\mu))}{4 n}\right](X-\eta(X) \xi) } \\
+ & {\left[\frac{(2(1-n)+\mu(4 n-1))}{4 n}\right] h X=0 . }
\end{aligned}
$$


In view of $(2.5),(5.3)$ takes the form

$$
h^{2} X=\left(\frac{2(1-n)+\mu(4 n-1)}{2(1-n)+n(2 k+\mu)}\right)(k-1) h X .
$$

Taking the trace of (5.4), we obtain

$$
\operatorname{trace}\left(h^{2}\right)=2 n(1-k)=0,
$$

which gives $k=1$. Thus $M$ becomes Sasakian. Hence we can state the following:

Theorem 5.1. $A(2 n+1)$-dimensional $(k, \mu)$-contact metric manifold $M$ with vanishing extended $\mathcal{M}$-projective curvature tensor is a Sasakian manifold.

From Theorem 5.1, we also state the following:

Corollary 5.1. A $(2 n+1)$-dimensional $N(k)$-contact metric manifold $M$ with vanishing extended $\mathcal{M}$-projective curvature tensor is a Sasakian manifold.

The unit tangent sphere bundle $T_{1} M$ equipped with the standard contact metric structure is a $(k, \mu)$-contact metric manifold if and only if the base manifold $M$ is of constant curvature $c$ with $k=c(2-c)$ and $\mu=-2 c([4])$. In case of $c \neq 1$, the unit tangent sphere bundle is non-Sasakian. Denote the unit tangent sphere bundle of a space of constant curvature $c$ with standard contact metric structure as $T_{1} M(c)$. Applying Theorem 5.1 to $T_{1} M(c)$, one can obtain

Corollary 5.2. In a $T_{1} M(c)$ if the extended $\mathcal{M}$-projective curvature tensor vanishes, then $c=1$.

Acknowledgment: The authors are thankful to referees for their valuable suggestions towards the improvement of the manuscript.

\section{REF E R E N C E S}

1. C. S. Bagewadi, D. G. Prakasha and Venkatesha: On pseudo projective curvature tensor of a contact metric manifold, SUT J. Math., 43, No. 1 (2007), $115-126$

2. D. E. Blair: Contact Manifolds in Riemannian Geometry, Lecture Notes in Math., 509, Springer-Verlag, 1976.

3. D. E. Blain: Two remarks on contact metric structures, Tohoku Math. J., 29 (1977), 319-324.

4. D. E. Blair, T. Koufogiongos and B. J. Papantoniou: Contact metric manifolds satisfying a nullity condition, Israel J. Math., 19 (1995), 189-214.

5. D. E. Blair, J. -S. Kim and M. M. TRipathi: On the concircular curvature tensor of a contact metric manifold, J. Korean Math. Soc., 42, (5) (2005), 883892. 
6. E. Boeckx: A full classification of contact metric $(k, \mu)$-spaces, Illinois J. Math., 44 (2000), pp. 212-219.

7. S. K. Chaubey and R. H. OJHA: On the $M$-projective curvature tensor on Kenmotsu manifolds, Different. Goem. Dynam. Syst., 12 (2010), 52-60.

8. J. T. Chо: A conformally flat $(k, \mu)$-space, Indian J. Pure. Appl. Math., 32 (2001), 501-508.

9. U. C. De, Y. H. Kim and A. A. Shaikh: Contact metric manifolds with $\xi$ belonging to $(k, \mu)$-nullity distribution, Indian J. Math., 47 (2005), 1-10.

10. U. C. DE, and A. SARKAR: On the quasi-conformal curvature tensor of a $(k, \mu)$ contact metric manifold, Math. Reports, Volume 14 (64), 2(2012), 115-129.

11. U. C. DE and P. PAL: On generalized $M$-projectively recurrent manifolds, Ann. Univ. Paedagog. Crac. Stud. Math., 13 (2014), 77-101.

12. U. C. DE and S. SAmui: E-Bochner curvature tensor on $(k, \mu)$-contact metric manifolds, Int. Electron. J. Geom., Volume 7 No. 1, (2014) pp. 143-153.

13. A. Ghosh, T. Koufogiongos and R. Sharma: Conformally flat contact metric manifolds, J. Geom., 70 (2001), 66-76.

14. R. H. OJнA: A note on the $M$-projective curvature tensor, Indian J. Pure Appl. Math., 8 (12) (1975), 1531-1534.

15. R. H. Олна: M-projectively flat Sasakian manifolds, Indian J. Pure Appl. Math., 17 (4) (1986), 481-484.

16. B. J. Papantoniou: contact Riemannian manifolds satisfying $R(\xi, X) \cdot R=0$ and $\xi \in(k, \mu)$-nullity distribution, Yokohama Math. J., 40 (1993), No. 2, pp. 149-161.

17. G. P. Pokhariyal and R. S. Mishra: Curvature tensor and their relativistic significance II, Yokohama Math. J., 19 (1971), 97-103.

18. S. TANno: Ricci curvatures of contact Riemannian manifolds, Tohoku Math. J., 40 (1988), pp. 441-448.

19. A. YILDIZ and U. C. DE: A classification of $(k, \mu)$-contact metric manifolds, Commun. Korean Math. Soc., 27 (2012), No. 2, 327-339.

Prakasha D. G.

Department of Mathematics

Karanatak University

Dharwad-580003, India

prakashadg@gmail.com

Kakasab Mirji

Department of Mathematics

Karanatak University

Dharwad-580003, India

mirjikk@gmail.com

Kakasab Mirji

PresentAddress: Department of Mathematics

KLS Gogte Institute of Technology, Jnana Ganga, Belagavi-590008 